
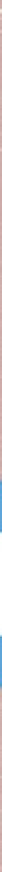

-
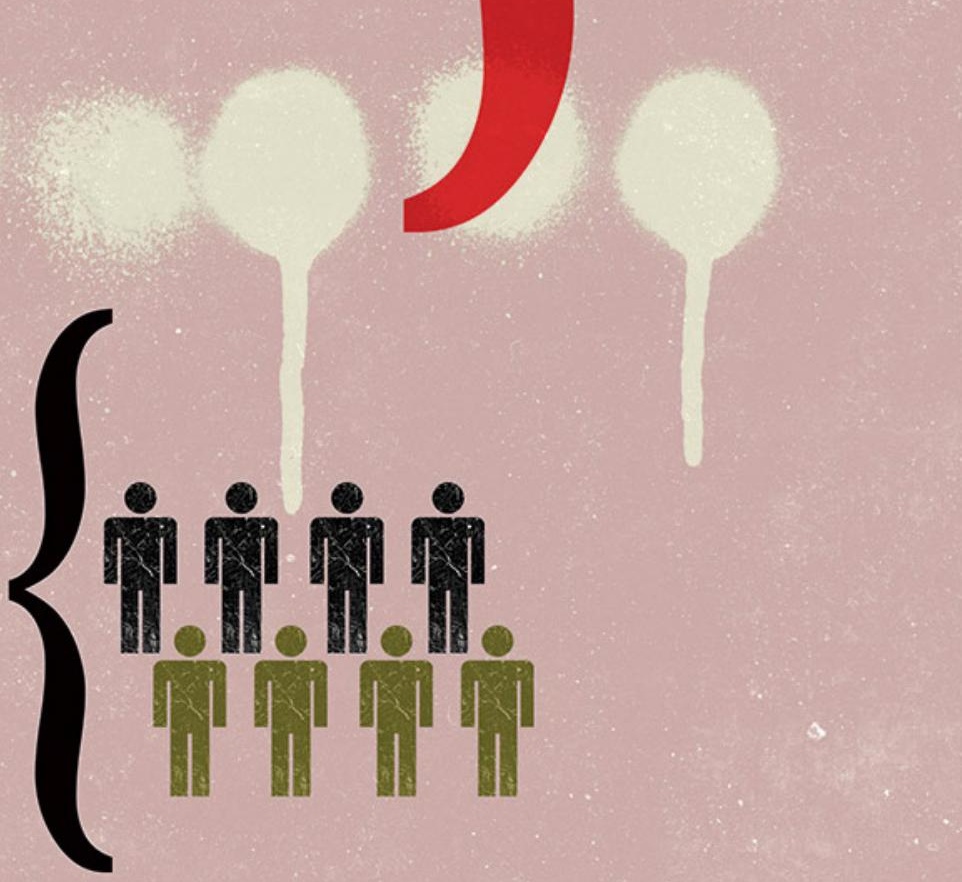


\title{
Actores, coaliciones y cambio institucional: la política social ante la reversión conservadora en Argentina y Brasil
}

\author{
Actors, coalitions and institutional change: \\ conservative shift and social policies in \\ Argentina and Brozil
}

Flarvio Goritớn*

\section{Resumen}

El artículo se propone analizar los cambios en las variedades de capitalismo de bienestar de Argentina y Brasil en el contexto de la transición política representada por la llegada al gobierno de coaliciones conservadoras, por medio de elecciones, en Argentina y a través de un irregular proceso de juicio político contra la presidenta Roussef, en el Brasil. El artículo analiza específicamente las políticas de seguridad social, las transferencias no condicionadas dirigidas a los "outsiders" y la regulación del mercado de trabajo. Por un lado, se presentan los cambios en las políticas públicas. Por otro, utilizando un marco teórico referenciado en las coaliciones, comunidades epistémicas y cambio institucional, buscamos entender las fuerzas dinámicas detrás de los cambios operados en materia de protección social.

Palavras-chave: Políticas sociales, bienestar, seguridad social.

\begin{abstract}
The article aims to analyze the changes and continuities in the varieties of welfare capitalism in Argentina and Brazil, in the context of the political transition represented by the arrival of conservative coalitions. by the government, through elections, in Argentina and through an irregular trial process politician against President Roussef, in Brazil. The article specifically analyzes social security policies, unconditional transfers directed to outsiders and labor market regulation. On the one hand, changes in public policies are presented. On the other, using a theoretical framework referenced in coalitions, epistemic communities and institutional change, we seek to understand the dynamic forces behind the changes in social protection.
\end{abstract}

Keywords: social policies, welfare, social security.

\footnotetext{
* Profesor adjunto de la UNILA, Universidade Federal da Integração Latino-americana. Investigador del INCT-PPED, Instituto Nacional de Ciência e Tecnologia em Políticas Públicas, Estratégias e Desenvolvimento. E-mail: flavio.gaitan@unila.edu.br.
} 


\section{INTRODUCCIÓN}

Luego de más de una década en que Argentina y Brasil, como otros países de la región, fueron gobernadas por coaliciones orientadas por ideas proclives a la intervención del Estado en la economía y la sociedad, ambos países han atravesado una transición en sus regímenes políticos representada por la llegada al poder de gobiernos ortodoxos; en Brasil, inicialmente con la irregular alternancia institucional que desplazó al Partido de los Trabajadores del poder (2016) y con la reciente elección de Jair Bolsonaro como presidente, en Argentina con la elección, por primera vez desde la reforma electoral de 1912, de un presidente liberal, que no pertenece a ninguno de los partidos tradicionales (PJ/UCR).

Teniendo en cuenta la mencionada transición política, el artículo se propone analizar los cambios en ambas variedades de capitalismo de bienestar, analizando, específicamente, las políticas de seguridad social, las transferencias no condicionadas dirigidas a los "outsiders" y la regulación del mercado de trabajo. Por un lado, se presentan los cambios en las políticas públicas. Por otro, utilizando un marco teórico referenciado en las coaliciones, comunidades epistémicas y cambio institucional, buscamos entender las fuerzas dinámicas detrás de los cambios operados en materia de protección social.

El artículo parte de considerar, siguiendo parte de la literatura sobre bienestar comparado (Wilensky, 1975, Arretche, 1995; Franco, 19960), la complementariedad entre regímenes productivos (Mercado) y variedades de capitalismo de bienestar. Las diferentes vertientes analíticas sobre bienestar en América Latina destacan la amplia heterogeneidad entre países y contextos (Cantú, 2016; Barba, 2003; Martínez Franzoni, 2007; Barba \& Lamelí, 2013; Lanzara \& Cantú, 2016), pese a lo cual se encuentran elementos en común: i. El empleo como una de las principales variables para analizar el bienestar en la región. Los mercados de trabajo de América Latina están definidos por el fenómeno denominado por Pinto (1970) dualidad o heterogeneidad estructural, relacionado con los ritmos diferenciales y no convergentes de productividad del trabajo.

Contradiciendo los postulados de la teoría de la modernización, la transición rural urbana no se expresó en una capacidad de creación de empleos formales. Teniendo en cuenta que los sistemas de protección tendieron a estar basados en el empleo formal, la dualidad económica genera desprotección para amplios sectores de trabajadores informales. La heterogeneidad estructural es producto del carácter de los mercados de de América Latina que, con escasas excepciones, tienen un carácter de economías primario-exportadoras, con escaso desarrollo industrial y un sector de servicios rezagado. Las variedades de capitalismo dependiente de América Latina se caracterizan por la amplitud de la economía informal y la incapacidad o desinterés de crear empleo formal y, iii. Teniendo en cuenta la caracterización de bienestar como la combinación de Estados, mercados y familias para enfrentar los riesgos sociales y el 
carácter de las variedades de capitalismo de América Latina que generan y reproducen la heterogeneidad estructural, el sector publico acabar siendo el factor estratégico para la generación de condiciones de bienestar.

\section{ACTORES, COALICIONES Y VARIEDADES DE BIENESTAR}

El Estado del arte sobre bienestar, en particular en los países de América Latina, puede ser clasificado en función de las variables explicativas sobre el momento de origen y consolidación de las políticas de protección social: i) la etapa de expansión y consolidación, desde el siglo XIX y hasta los años 1980; ii) el periodo de reformas neoliberales entre los años 1980 y 1990 y, iii) la fase de expansión segmentada desde finales de los años 1990 hasta el presente (Antía, 2018). En esta sección, nos centramos en los factores explicativos de la tercera fase, cuya característica distintiva es la inclusión de los "outsiders" (Garay, 2016), para lo cual se han desplegado, en la mayoría de los países de la región, dos mecanismos principales: pensiones no contributivas y transferencias monetarias condicionadas. La literatura que explica dichos cambios tiende a destacar diferentes factores.

Un conjunto de trabajos recupera la importancia de las presiones externas, de diferentes fuerzas. Algunos autores destacan la difusión de políticas, afirmando que las administraciones (nacionales o subnacionales) tienden a adoptar políticas públicas que han sido exitosas en otros contextos (Borges Sugiyama, 2011; Weyland, 2006). En este sentido, la capacidad de expertise de las organizaciones internacionales suele ser identificado como un factor relevante al momento de explicar la amplia adopción de este tipo de programa en diferentes países (Desai, 2007). Una explicación alternativa enfatiza los efectos positives del boom de commodities y el contexto externo sobre las posibilidades de implementar políticas de redistribución (Rossi, 2015). Por último, las presiones derivadas de la globalización (Seguro Ubiergo, 2007).

Una segunda vertiente explicativa explora la importancia de la democracia para a redistribución (Segura Ubiergo, 2007; Mares \& Carnes, 2009). Así, los autores resaltan el rol de la democracia en dar forma a la arena política y la incorporación de demandas sociales (Haggard \& Kaufman, 2008), la relación entre democracia y provisión de servicios sociales (Pribble, Huber \& Stephens, 2009), el aumento en el gasto social desde la transición democrática (Hanlon, Barrientos \& Hulme, 2010) y, de modo particular, la importancia de la democracia para aumentar la competencia electoral y la capacidad de los actores para influir las políticas (Dion, 2009).

La mayor competencia electoral es recuperada como un elemento central para explicar la expansión de las políticas sociales, al menos en dos sentidos. En primer lugar, se le asigna una importancia estratégica a apoyo electoral derivado de la expansión de las políticas. Diferentes autores afirman que existe una relación directamente proporcional entre mayor competencia electoral y tendencia de los partidos a crear o ampliar programas sociales (Garay, 2016; Desai, 2007; Hanlon, Barrientos \& Hulme, 
2010). De hecho, diferentes estudios examinan la asociación entre protección social a los outsiders y apoyo electoral. En algún sentido, la expansión de las políticas sociales es considerada un instrumento para ganar elecciones o conservar apoyo político. En segundo lugar, apoyo político a través de la movilización popular. Etchemendy (2017) encuentra diferentes tipos de alineamiento político entre gobiernos, sindicatos y movimientos sociales en los países de América Latina. Garay (2016, p. 24-25) señala que "incumbents expanded social policy when faced with high levels of electoral competition for the vote of outsiders and/or with large-scale social mobilization ...."

La idea de que existe una relación entre expansión de las políticas sociales y mayor competencia electoral se relaciona, al menos parcialmente, con la visión de los partidos políticos como organizaciones cuyo principal objetivo es ganar elecciones. Sin embargo, otras explicaciones resaltan que no se trata apenas de mayor apoyo electoral o de movilización. En ese sentido, señalan el papel de las ideas. Los funcionarios (policymakers) formulan e implementan políticas públicas no solo en la busca de incentivos o recompensas políticas sino, también, por compromisos con ciertas ideas (Borges Nagayama, 2012). En este sentido, las creencias e ideologías deben ser tenidas en cuenta como factor explicativo.

Las categorías de actores que forman parte del ciclo de políticas de expansión de la protección social son consideradas desde diferentes perspectivas. Un grupo de analistas se centra en los tipos de actores políticos, en particular, los movimientos sociales y su capacidad de interpelación al poder político (Garay, 2016; Etchemendy, 2017), el papel de los sectores populares (Pribble 2013), las elites (Filgueira, 2005), los líderes políticos (Desai, 2007; Boschi \& Gaitán, 2016) y la burocracia (IPEA, 2015). También es considerada la naturaleza de los partidos políticos (Pribble, 2013). En este sentido, un grupo de autores reconoce la importancia de los gobiernos de izquierda o centro-izquierda (Huber \& Stephens, 2012; Pribble 2013). Desde esta perspectiva, las coaliciones de clase dan forma a diferentes variedades de medidas de protección social (Holland \& Schneider, 2016).

Así, la competencia electoral explica, hasta un punto, la expansión de la política social, pero no contribuye a entender la variedad de objetivos, programas y recursos. Las ideas deben ser tenidas en cuenta. Para explicar su importancia sobre las políticas públicas, se han formulado diferentes modelos teóricos que buscan entender por qué los gobiernos adoptan una política en detrimento de otras, como agenda setting, advocacy coalitions and policy arenas. Tanto el modelo de coaliciones de defensa y de arenas de política reconocen la importancia del nivel ideacional, afirmando que las ideas impactan sobre las políticas públicas (Hall, 1992; Weir, 1992; Boschi and Gaitán, 2016; Haas, 1992,) por diferentes razones. La explicación es simple: los actores portan ideas que se plasma en políticas.

Considerando esta breve discusión teórica, adoptamos como hipótesis de trabajo las siguientes hipótesis: 1. En un contexto de mayor competencia electoral, aumentan los incentivos para incrementar las políticas de protección social. Esto sucede porque 
los líderes políticos en posición de gobierno tienden a priorizar la protección social para lograr fidelidad electoral (Garay, 2016, Dion, 2009). Esta situación se puede dar en diferentes niveles de gobierno (Niedzwiecki, 2016). La mayor competencia electoral facilita la expansión de las políticas pero no explica la variación de políticas en diferentes contextos. A pesar de la mayor competencia electoral, los programas sociales expresa una amplia variación en población protegida, mecanismos, objetivos y recursos. Así, 2. La variación en las formas de protección social se explica por la presencia de coaliciones ideacionales que portan innovaciones en el contexto de coyunturas críticas. En nuestra perspectiva, las diferentes respuestas en material de protección social es resultado de presiones que pueden resultar de los movimientos sociales (Garay, 2016), partidos políticos (Dion, 2009) o la ideología de los actores estratégicos (Huber \& Stephans, 2012). Asumimos que no se trata apenas de presiones populares o de incorporación de actores de movimientos sociales en el aparato administrativo del Estado. Es necesario crear coaliciones de ideas para impulsar ciertas políticas en detrimento de otras.

\section{METODOLOGÍA Y SELECCIÓN DE CASOS}

Este trabajo consiste en un estudio cualitativo de N pequeña. Como afirman King, Keohane \& Verba (1994: 139) en estudios cualitativos"(...) la selección (de casos) debe ser realizada de modo intencional, consistente con los objetivos de investigación y las estrategias". Para seleccionar los casos, se ha adoptado la estrategia de casos más similares (Przeworski \& Teune, 1970), que considera que los casos seleccionados deben asumir valores similares en un conjunto de variables consideradas constantes, encontrando al menos una diferencia significativa en la variable explicativa. Esta estrategia tiene el mérito de reducir el número de variables relevantes para encontrar una explicación al fenómeno de interés. En nuestra perspectiva, como deriva de la sección anterior, las diferencias en la adopción de programas se relacionan con las coaliciones ideacionales. Para poder someter a prueba la hipótesis adoptamos el método histórico comparada, que busca explicar las causas y consecuencias históricas focalizando en la observación de procesos (Mahoney, 2012). Así, otorga importancia explicativa a "(las) causas-de efectos; (tratando de) explicar por qué ciertas causas tienen resultados específicos" (Mahoney, 2010: 132).

\section{CAMBIOS Y RUPTURAS EN EL SISTEMA DE PROTECCIÓN SOCIAL EN ARGENTINA}

Argentina conformó su sistema de protección social a lo largo de un período que comienza en el siglo XIX con el establecimiento de la obligatoriedad de la educación y se extiende con fuerza desde comienzos del siglo XX con la creación de diferentes cajas de seguridad social para diferentes categorías ocupacionales y legislación social 
que regulaba las condiciones de empleo, ampliando la cobertura durante la posguerra con la unificación de las cajas, la creación del sistema asignaciones familiares y un avance en la formalización de la relación de trabajo, entre otros elementos. El modelo de protección se basaba, básicamente, en la figura de hombre trabajador formal. Se consolidó una asociación entre empleo y seguro social, por diferentes categorías ocupacionales, con un fuerte sesgo de fragmentación de beneficios. Al mismo tiempo, algunas prestaciones asistenciales para las personas vulnerables. En la medida que entre la salida de la crisis de los años treinta y hasta comienzo de los años sesenta, el país contó con bajo desempleo, esa particular combinación permitió una ampliación de la cobertura formal y una situación próxima a un Estado de bienestar informal (como definido por Barrientos, 2004).

El periodo iniciado durante la dictadura militar y consolidado durante la administración Justicialista de Carlos Menem (1989-2001) representó una ruptura con la modalidad de capitalismo estado-céntrica protegida. Las reformas ortodoxas adoptadas por el período modificaron las bases de funcionamiento de la economía y generaron un aumento significativo del desempleo. En materia de políticas sociales y laborales se produjeron profundas reformas tendientes a la flexibilización y la adopción de políticas subsidiarias y residuales. La lógica subsidiaria se asentó en un esquema "Estado para pobres, mercado para quienes pudieran pagar". Las diferentes medidas representaron una ruptura de los pilares de la protección social que se fue construyendo a lo largo del siglo XX, llegando a su punto culmine en la segunda posguerra'.

Durante la primera década del siglo XXI se avanzará en la conformación de un sistema de protección que busca igualar los derechos de los trabajadores formales a las protecciones de existentes para los trabajadores formales en ese sentido a través de los grandes mecanismos los programas de transferencias condicionadas y la expansión de las jubilaciones ex post se produjo una ampliación de derechos para los sectores históricamente marginados.

A partir de 2002 se revierte parte del legado neoliberal, con la adopción de una serie de medidas, en tres frentes: políticas condicionadas, expansión de la protección a los adultos mayores con una camino alternativo al de las pensiones no contributivas y re-regulación de las relaciones laborales. Inicialmente con el programa de Plan Jefes y Jefas de Hogar desocupados, implementado durante la crisis de 2002.

La recuperación económica y la lógica de "inserción por el empleo" llevaron a la coalición gobernante a modificar el Plan Jefes, iniciando un traspaso hacia otros programas sociales o hacia estrategias de capacitación laboral. A inicios del 2006 el MTEySS puso en marcha el Seguro de Capacitación y Empleo (SCyE; creado en octubre de 2004 por Decreto 1506) para facilitar la reorientación del Programa Jefes y Jefas de Hogar. Se trataba de un seguro de base no contributiva destinado a personas desocupadas, en particular los beneficiarios del PJJH. Esta transición implicó un incremento de la asignación mensual del PJJH (a 225 pesos -un poco más de 
50 dólares estadounidenses de ese período), es de duración limitada, estableciendo una permanencia máxima de dos años, aunque contó con dos ventajas en relación a otros programas: por un lado, el período fue considerado como activo para el computo de la jubilación de los beneficiarios; por otro lado, la percepción no era incompatible con un trabajo formal, lo que no desincentivó el potencial ingreso al mercado formal de empleo por parte de los beneficiarios. El Seguro de Capacitación y Empleo se fundamentaba en la necesidad de fortalecer la actual Red de Oficinas de Empleo Municipales²; el desarrollo de las calificaciones de la población trabajadora a través de procesos de certificación de competencias laborales y la promoción de una oferta de formación profesional de calidad; y el desarrollo de un conjunto de herramientas para la promoción del empleo y la inserción laboral con base local o territorial.

Desde el año 2005 y bajo la órbita del Ministerio de Desarrollo Social, se puso en marcha el Programa "Familias por la inclusión social", cuyo objetivo fundamental residía en promover la protección e integración social de las familias en situación de vulnerabilidad o riesgo social desde la salud, la educación y el desarrollo de capacidades (MDS, 2005) El Plan incorporaba a los antiguos beneficiarios del Plan Jefas y Jefes mediante traspaso voluntario y consistía en la entrega de una prestación monetaria a las familias por cada niño menor de 19 años y por embarazada, cuya depositaria y titular es la madre. El monto era calculado en proporción al tamaño de la familia a partir de un mínimo de 100 pesos (unos treinta dólares del momento) mensuales por hijo o mujer embarazada y 25 pesos por cada hijo adicional, hasta un máximo de 5 hijos y de 200 pesos por familia. Como contrapartida, la madre o mujer responsable debía comprometerse a demostrar contraprestaciones de salud y educación ${ }^{3}$.

En materia previsional, la Ley 26.425 creó el Sistema Integrado Previsional Argentino, eliminando el régimen de capitalización individual y fortaleciendo los recursos públicos a partir de la creación del Fondo de Garantía de Solidaridad, compuesto por una combinación de aportes de los trabajadores y los empresarios y contribuciones tributarias generales ${ }^{4}$, aumentó significativamente en los últimos años, pasando de menos de cien mil millones de pesos a más de quinientos mil millones en 7 años ${ }^{5}$. Por otro lado, fue aprobada por Ley 25.994 una moratoria, el Plan de Inclusión Previsional, para integrar al sistema a aquellos trabajadores que no contaban con los años de aporte exigidos por Ley (60 años las mujeres, 65 los hombres) o que, teniendo aportes, pero no la edad mínima requerida, no podían acceder a un beneficio. Durante el período laboral, de alto aumento del desempleo, los requisitos mínimos para acceder a una jubilación habían aumentado severamente, pasando de 10 a 30 años. La combinación de alto desempleo, privatización del sistema de administración de los fondos previsionales y de mayores requisitos implicó la exclusión de una importante parte de la población del beneficio previsional. Con el sistema de moratoria, que posibilitó el pago en cuotas para aquellos sin ningún tipo de aportes o con aportes insuficientes, representó una solución original que amplió la cobertura de jubilación de los adultos mayores. Las altas pasaron de 22.000 en 2005 a 640.000 en 2006, 
1.050.000 en 2007, 290.000 en 2008 y 310.000 en 2009, superando los 2 millones de nuevos beneficiarios (ANSES, 2010). En 2014 hubo una segunda moratoria, que sumó casi medio millón de nuevos beneficiarios, llevando la cobertura al 95,8\% de los adultos mayores.

En materia de empleo, el modelo implementado a partir de 2003 declaró prioritario una nueva concepción del trabajo como articulador entre la esfera económica y social, como base de la cohesión, como fuente de dignidad y como factor constitutivo de la ciudadanía (Novick y Tomada, 2007). Este proceso fue posible por el contexto internacional favorable, por la implementación de políticas heterodoxas en lo económico y por el papel activo del Estado en la coordinación y orientación económica. Una serie de cambios merecen ser destacados. En primer lugar, se promovió la negociación colectiva, reactivando el Consejo del Salario, paralizado durante el período neoliberal. De hecho, las negociaciones pasando de 348 convenios que alcanzaron a 1,2 millón de trabajadores en 2004 a 1964 convenios que protegieron a 4,3 millones de asalariado. ${ }^{6}$ Se derogó la Reforma Laboral aprobada en el año 2000 durante la Administración de la Alianza y se sancionaron normas limitando la amplia desregulación de las relaciones laborales: la Ley 25.520 limitó el a 3 meses el período de prueba; la Ley 26.476 estableció un régimen de regularización del empleo no registrado; la Ley 18.441 equiparó la jornada de trabajo de los trabajadores rurales al resto de las actividades (8 horas diarias y 48 semanales). El restablecimiento de las paritarias, la caída del desempleo (pasó de 20,5\% en 2003 a 7,3\% en 2008, a pesar de que la PEA aumentó significativamente) y la postura de la coalición de gobierno generaron un aumento continuo del salario mínimo, el cual había estado fijo durante el período neoliberal. Medido en pesos corrientes fue de 450 en 2004, 630 en 2005, 800 en 2006, 980 en 2007, 1240 en 2008, 1440 en 2009, 1740 en 2010, 2300 en 2011, 2670 en 2012, 3300 en $2013^{7}$.

En noviembre de 2009, un mes más tarde de la derrota de la coalición gobernante en la provincia de Buenos Aires ( $y$, particularmente, en un espacio considerado "propio" como es el segundo cordón del Conurbano ${ }^{8}$ ) fue implementado, por medio del Decreto de Necesidad y Urgencia 1602/09 del Poder Ejecutivo Nacional la "Asignación Universal por Hijo para Protección Social" . La medida está destinada "a aquellos niños, niñas y adolescentes residentes en la República Argentina, que no tengan otra asignación familiar prevista por la presente ley y pertenezcan a grupos familiares que se encuentren desocupados o se desempeñen en la economía informal" (Decreto 1602/09).

La AUH consiste en una transferencia monetaria mensual, no retributiva, por hijo - hasta un máximo de 5 hijos - a padres o madres que tengan a cargo hijos menores de 18 años. Los beneficiarios deben ser argentinos nativos o naturalizados con una residencia legal en el país mínima de tres años, no estar percibiendo otra asignación familiar y pertenecer a hogares donde los padres sean desocupados, monotributistas sociales ${ }^{10}$, trabajadores no registrados o del servicio doméstico y perciban un ingreso 
por debajo del Salario Mínimo Vital y Móvil. El programa tiene la particularidad de que el veinte por ciento del monto del beneficio es pago una vez al año, luego de ser comprobados las condicionalidades de asistencia escolar y cumplimiento del calendario de vacunas obligatorio. El objetivo principal del programa es equiparar el ingreso de los niños cuyos padres no están incorporados al mercado de trabajo formal, con los de los niños cuyos padres, por estar empleados formalmente, reciben las sumas correspondientes establecidas por el Régimen contributivo de Asignaciones Familiares regulado por Ley 24.714 (Arcidiácono, 2016).

En agosto del mismo año se anunció el "Programa de Ingreso Social con Trabajo" ("Argentina Trabaja"), el cual declaraba como principal objetivo la promoción del desarrollo económico y la inclusión social, buscando crear nuevos puestos de trabajo genuinos, con "igualdad de oportunidades", apuntalando el trabajo organizado y comunitario e incentivando e impulsando la formación de organizaciones sociales de trabajadores. El plan, creado por Decreto 1067/09 (Resolución 3182 del MDS) está destinado exclusivamente a quienes no tienen empleo ni tampoco ningún tipo de subsidio directo del Estado (excepción hecha de la AUH, que es compatible con este programa). El mecanismo para poder ser beneficiario es formar parte de una cooperativa de trabajo, que realizan tareas bajo la coordinación de un Ente Ejecutor que pude ser tanto un municipio, una provincia o el organismo público que articula con cooperativas, el INAES. El Plan y el presupuesto asignado fueron ampliados" progresivamente, pasando en un breve espacio de tiempo de una estimativa inicial de 70.000 beneficiarios a poco menos de 200.000. También en 2009 fue relanzado el REPRO, el Programa de Recuperación Productiva, creado por la Resolución del Ministerio de Trabajo, Empleo y Seguridad Social № 481/02 en el marco de la Emergencia Ocupacional Nacional y que volvió a tomar fuerza en 2009, con el telón de fondo de la crisis capitalista del centro económico, con sucesivas prórrogas de vigencia progresivamente, pasando en un breve espacio de tiempo de una estimativa inicial de 70.000 beneficiarios a poco menos de 200.000 .

\section{CENTRALIDAD DEL MERCADO Y POLÍTICAS COMPENSATORIAS}

La coalición Cambiemos llevó a cabo una política de "normalización" de las variables económicas, implementando una serie de reformas (apertura comercial, liberalización del mercado de cambios, equiparación de las tarifas públicas a valores del mercado, devaluación de la moneda) que tuvieron impacto sobre los niveles de actividad económica, el costo de vida y el desempleo. Elementos que deben ser tenidos en cuenta para analizar la orientación de las políticas sociales y su influencia sobre la variedad de capitalismo de bienestar.

A poco de comenzar la gestión, el gobierno implementó una política que se denominó reparación histórica para los jubilados y jubiladas por medio del cual creó un programa voluntario para que adhirieran todos aquellos que estuvieran llevando 
adelante juicios contra la ANSES por cálculos erróneos de sus haberes. La norma 27260/16 buscaba así poner fin a un problema histórico del sistema de seguridad social argentino, caracterizado por la alta litigidad previsional, a la que la norma considera en emergencia. En segundo lugar, se creó una pensión no contributiva, denominada Prestación Básica Universal (PBU). Según consta en la página de internet de la ANSES, se trata de una asignación no contributiva vitalicia y que busca incluir a los adultos que tenga 65 años o más que no cuenten con ningún ingreso. Entre los requisitos para que las personas puedan acceder a la pensión universal se encuentran no ser titular de ningún otro programa social (tanto de carácter contributivo como no Contributivo) y ser argentino o residente con 20 años o naturalizado con 10 años de residencia.

La PUAM representa el $80 \%$ de la jubilación mínima y no es heredable, estableciendo una distinción entre jubilados y pensionados y beneficiarios de la PUAM. A pesar de que su impacto es limitado, teniendo en cuenta que las moratorias llevaron la cobertura previsional al $97 \%$ de los adultos en edad de jubilarse, impone una lógica asistencial y contribuye a la segmentación de derechos. Con esta medida, el gobierno se aleja de la práctica de moratoria, que reconoce el trabajo informal y doméstico y equipara los derechos de trabajadores formales e informales y aproxima la política de seguridad social orientada a los "outsiders" a la lógica focalizada y asistencialista que predomina en un amplio conjunto de países de la región que otorgan pensiones no contributivas. En ese sentido, este año fue introducida la obligatoriedad de realizar una evaluación socio-económica.

En tercer lugar, el gobierno promovió una reforma previsional, sancionada en la ley 27426/17 y promulgadas por el PEN 1096/17, que modifican la herencia del Sistema Integrado de Jubilaciones y Pensiones (SIPA) creado durante la administración de la presidenta Fernández. La norma, aprobada en un clima de hostilidad y fuertes críticas de sectores de la oposición, modifica el cálculo de los aumentos establecidos en el artículo 32 de la ley 24.241/09. La normativa aprobada en 2009 establecía que debía llevar se a cabo una movilidad de los haberes jubilatorios dos veces por año, resultado de la combinación de un índice de aumentos de los salarios y otro de ingresos de la ANSES. Con la reforma promovida por el presidente Macri la movilidad pasa a estar relacionada con un índice combinado de la inflación (Índice precios al consumidor que elabora el Instituto Nacional de Estadísticas y Censos) y un índice que surge de la remuneración imponible media de los trabajadores estables (denominado RIPTE). La formula, contemplada en el anexo de la normativa aprobada, divide el peso en $70 \%$ para el promedio del aumento de precios y $30 \%$ para el aumento de las remuneraciones. Al mismo tiempo, establece que en lugar de dos aumentos anuales pasarán a ser cuatro, en los meses de marzo, junio, septiembre y diciembre de cada año. Además, se garantiza a los jubilados y pensionados que completaron 30 años o más de servicios con aportes (sin adherir a moratorias previsionales) un cobro extra que permita alcanzar en un $82 \%$ el valor del Salario Mínimo Vital y Móvil. ${ }^{11}$. Aprobada 
y reglamentada a finales de 2017, la norma comenzó efectivamente a ser aplicada en marzo de $2018^{12}$.

Por último, el Ministerio de Desarrollo Social intentó avanzar en la eliminación de pensiones no contributivas contempladas por Ley. El sistema de protección social argentino incluye las pensiones no contributivas reguladas por ley y que contempla a madres con siete hijos y personas de 70 años o más y discapacitados. Esgrimiendo una narrativa de transparencia y un supuesto uso indebido del sistema de pensiones, el gobierno aplicó el Decreto 432/97 y dio de baja 170 mil pensiones. En un segundo momento, apeló el fallo de la Justicia que lo intimaba a restablecerlas.

En materia de asignaciones familiares, continua la fragmentación entre programas dirigidos a hijos de trabajadores formales y aquellos orientados a hijos de informales. Pese a esto, el gobierno amplió la cobertura de la asignación universal por hijo de manera de poder incluir a los hijos de cuentapropistas, en particular aquellos de menores ingresos, que no estaban contemplados en el monotributo social. Con esta medida, se produjo un aumento en el número de niñas/os contemplados. Por otro lado, la Ley de movilidad generó aumentos en el haber de los montos, aunque inferiores a la inflación ${ }^{13}$.

Los planes de empleo autogestionado y cooperativo pasaron por una primera etapa en la que fueron reorientados hacia la capacitación y el apoyo a la reinserción laboral, aumentando el control sobre la eficiencia y focalización del gasto en los sectores más vulnerables y un corrimiento del papel de las cooperativas (Programa de Inclusión Social con Trabajo, Argentina Trabaja) y la formación integral y el énfasis en actividades sociales y comunitarias (Ellas Hacen). De todos modos, el programa aumentó en número de 107 mil en diciembre de 2015, 175 en diciembre de 2017, en parte por la sanción de la Ley de Emergencia Social.

En un segundo momento, en febrero de 2018 el gobierno avanzó en una reconfiguración del programa, estableciendo el traspaso de los beneficiarios del Argentina Trabaja y el Ellas Hacen al recién creado programa Hacemos Futuro, con el supuesto interés de brindar autonomía de la persona, centrándose en la idea de "terminalidad educativa" y "educación integral". El traspaso y la idea declarada de potenciar la "autonomía" representaba, al mismo tiempo, el menor espacio de participación de las cooperativas de trabajadoras y trabajadores desempleados en la gestión de los programa, avanzando en una lógica de "individualización" de la protección. El traspaso de los programas Argentina Trabaja y Ellas Hacen al recién creado programa Hacemos Futuro fue producto de un supuesto análisis por parte de los funcionarios de gobierno. El programa, de acuerdo a los considerandos de la Resolución 151/2018 se centra en la población vulnerable con el objetivo de posibilitar la "terminalidad educativa y formación integral". Al mismo tiempo, avanza sobre el control de los beneficiarios (encuestas socio-educativas, presentación de certificados que acrediten la participación en cursos y capacitación, Por otro lado, desinterés por las 
prácticas de las cooperativas (que no funcionaran no es justificativa). Las cooperativas dejan de ser un requisito. Las organizaciones dejan de gestionar los planes.

La ambigüedad del gobierno consiste en que, al tiempo que reorganiza el programa, se enmarca en una negociación con las organizaciones sociales demandantes de una Ley que declarase la emergencia social, que crease un millón de puestos de trabajo, un aumento en las asignaciones y el establecimiento de un salario anual complementario. Producto de una fuerte disputa entre las organizaciones del campo social (en particular las organizaciones de trabajadores desocupados) y sectores del oficialismo, el gobierno cedió a las demandas sociales y la norma fue aprobada en Diciembre de 2016 y promulgada en febrero de 2017, asignando un presupuesto de 25 mil millones de pesos para la emergencia y creando una serie de innovaciones institucionales: i. El Consejo de la Economía Popular y el Salario Social Complementario (CEPSSC), con una función equivalente a la de una paritaria de social de los sectores informales, conformado un/a representante del Ministerio de Trabajo, Empleo y Seguridad Social de la Nación ${ }^{14}$, un/a representante del Ministerio de Desarrollo Social de la Nación y tres representantes de organizaciones sociales debidamente matriculadas en el Registro de Organizaciones Sociales de la Economía Popular y empresas gestionadas, que fuera creado por la Resolución 32/2016 del Ministerio de Trabajo, Empleo y Seguridad Social; ii. La figura de salario social complementario, estbleciendo el valor en 4000 pesos, 50\% del salario mínimo de 8060 al momento de la negociación de la Ley, considerando que es la brecha entre la percepción de ingresos de los informales y el salario mínimo y, iii. El Registro de Trabajadores de la Economía Popular (RENATREP), con la intención de avanzar en un traspaso de planes sociales al pago de un salario social complementario, otorgando carácter de trabajadores de la economía popular a trabajadores desocupados e informales.

A pesar de que, por interés propia o empujado por la presión de las organizaciones sociales, el gobierno no generó grandes cambios en materia de políticas sociales, sin ser original, mantuvo los programas pero implementó cambios empujando la esencia hacia el mercado, la empleabilidad y la capacitación. El gasto en prestaciones sociales aumentó (sobre el gasto total representó 48,4\% en 2016, 56,4\% en 2017 y 60,8\% en 2018) pero su efecto se ve socavado por los indicadores del mercado de empleo y la distribución primaria del ingreso. Aumentó el desempleo (de 9,1\% en el primer trimestre de 2018 a 10,1\% el primer trimestre de 2019), en forma concomitante a una caída en la tasa de actividad y la tasa de empleo ${ }^{15}$. También aumentó el subempleo (9,8\% en 2018 a 11,8\% en 2019).

El impacto del desempleo y el subempleo se vio potenciado por una serie de políticas del gabinete. En primer lugar, la decisión de limitar las paritarias a un ritmo sensiblemente inferior al de la inflación pasada. De hecho, la intervención del Ministerio de Trabajo buscó que los aumentos no excedieron el $25 \%$ anual, en un año en que la inflación llegó al 40\%. Esta estrategia es funcional a la retórica oficial que busca disciplinar a los trabajadores, para alcanzar relaciones laborales pacíficas 
("no poner palos en la rueda", en palabras del presidente). De hecho, el propio titular del Ejecutivo se enredó en una discusión pública con abogados laboristas y sindicalistas por su apelo a "terminar con la industria del juicio"'!16, respaldada por el empresariado" La retórica y decisiones oficiales expresan la visión de asociar salarios a costo laboral, en una estrategia que se aproxima a la filosofía neoliberal de considerar las relaciones laborales como un tema "privado" entre empleador y empleado. En ese sentido se ubican las estrategias de avanzar en acuerdos salariales ajustados por "productividad", un camino adoptado por economías con modelos ortodoxos como México.

En segundo lugar, la quita de los subsidios al transporte y los servicios públicos. Estos aumentos, llevados a cabo por decreto y sin la realización de las audiencias públicas contempladas por Ley, fijaron un "tope" de $400 \%$ para los servicios residenciales y $500 \%$ para las industrias, una vez que los aumentos iniciales, superiores a esos montos, llevara a la judicialización del proceso. Los aumentos impactaron especialmente en los sectores populares.

En tercer lugar, la inflación, en especial de alimentos ${ }^{18}$. En Argentina, hay una serie de características que implican que variaciones en la política cambiaria impacten en el precio de los alimentos. Por un lado, el país exporta alimentos que consume, lo que genera que, ante la apertura de exportaciones y la eliminación de las retenciones, se acabe "importando inflación". Por otro lado, la estructura económica oligopolizada combinada con una actitud supuestamente "defensiva" de un empresariado rentista dificulta bajas los precios de los productos de consumo.

En cuarto lugar, un formato de políticas sociales que parece más cercano a la visión asistencialista que a un enfoque de derechos, agravada por "problemas de gestión"19. A pesar que no se han desactivado los pilares de políticas, la narrativa se basa en la asistencia como paliativo frente a una situación de desempleo entendida como anómala y transitoria ${ }^{20}$.

\section{POLÍTICAS SOCIALES DURANTE EL PERÍODO POST-NEOLIBERAL EN BRASIL}

Diversos autores afirman que en el Brasil las políticas sociales han estado, en gran medida, relacionadas de modo subalterno a los proyectos de desarrollo económico (Vasconcelos, 2016; Draibe, 1993). Los derechos de ciudadanía social se enmarcaban dentro de la figura definida por Santos (1979) como ciudadanía regulada, en referencia a un padrón de acceso a derechos directamente dependiente de la inserción en el mercado de trabajo, con protección diferencial en virtud de las categorías ocupacionales (Bichir, 2016). Existe un relativo consenso en identificar al proceso de reforma constitucional de 1988 como la piedra basal del sistema de protección social del Brasil democrático. LA Constitución estableció un capítulo específico para los derechos sociales (salud, asistencia, previsión social, educación y derecho a la vivienda). En ese sentido, se consolidó un segundo movimiento de transición. La 
dictadura militar estableció un padrón de políticas de carácter regresivo (Draibe, 1993); el período democrático consolidó, lenta, paulatina y constantemente un proceso de universalización de derechos (Bichir, 2016).

Las políticas de protección se ampliaron desde la sanción de la Constitución Federal de 1988 y de leyes reglamentarias de medidas establecidas en la Carta Magna, creciendo particularmente a partir de 2002, desde la llegada al poder de la coalición liderada por el Partido de los Trabajadores y la adopción de una serie de políticas y de reformas institucionales (creación del Ministerio de Desarrollo Social y Combate al Hambre, implementación de consejos de participación, Registro único de beneficiarios, entre otras). En relación al sistema previsional, el sistema brasileño es de carácter contributivo y afiliación obligatoria, existiendo beneficios de pensión para riesgos de invalidez y fallecimiento, además de otros auxilios relativos a maternidad, desempleo, enfermedad y accidentes de trabajo (Lanzara, 2016). Los niveles de cobertura se encuentran por encima del $80 \%$. En otra orden, garantizada por la Constitución, existen programas de asistencia social que contemplan beneficios para grupos en situación de riesgo, en particular, el Beneficio de Prestación Continuada, una renta para personas mayores de 65 años o con discapacidad, con renta per cápita inferior al $25 \%$ del salario mínimo (Lanzara, 2016).

En materia de asistencia social, inicialmente el presidente Lula anunció el Programa Fome Zero, cuya meta consistía en erradicar el hambre en un período de cuatro años y reducir los niveles de desnutrición hasta 2015. El programa sería luego reemplazado por el Bolsa Familia, creado por Ley no 10.836/2004 y reglamentado por el Decreto 5.209/2004, El programa condensó otros programas existentes, creados durante las administraciones de Fernando Cardoso: Bolsa Escola que era gestionado por el Ministerio de Educación, el Bolsa Alimentación, a cargo de Ministerio de Salud, el Cartão Alimentação y el Auxílio Gas, responsabilidad del Ministerio de Minas y Energía ${ }^{21}$. La unificación de los cuatro programas en el Bolsa Familia buscaba superar problemas de coordinación, superposición de estructuras administrativas y fragmentación en la cobertura poblacional.

Formulado e implementado de manera bastante centralizada desde el gobierno nacional ${ }^{22}$, con el concurso de los municipios al momento de realizar el registro en el Cadastro de Beneficiarios, el Bolsa Familia ${ }^{23}$ Ilegó a "proteger" más de 13 millones de familias y 52 millones de personas. El programa consiste en una transferencia monetaria condicionada a familias en situación de pobreza, que establece una especie de contratos con los beneficiarios, por el cual se establecen deberes entre los cuales resalta la asistencia escolar (mínimo de 85\% hasta los 15 años de edad) y el control sanitario a través de las carteras de vacunación. La transferencia monetaria contempla dos componentes: uno básico, destinado a las familias extremamente pobres, que en la actualidad es de $\mathrm{R} \$ \mathbf{8 5}$, otro variable, para familias en situación de pobreza o extrema pobreza, que tengan miembros niños y adolescentes de hasta 15 años, mujeres embarazadas, con valor individual de $\mathrm{R} \$ 39$ y hasta $\mathrm{R} \$ 1$ 195. Si los hijos son 
adolescentes de 16 o 17 años, el valor es de R\$ 46 (hasta R\$ 92). El número máximo que una familia puede recibir es de 5 beneficios variables y con un valor máximo, combinando ambos beneficios, de $\mathrm{R} \$ 372$, en un país donde el salario mínimo es de $\mathrm{R} \$ 937$. En los dos primeros años del programa cubría a 6.6 millones de familias y representaba casi un cuarto del gasto del país en seguridad social. En sus primeras fases, las familias en situación de pobreza eran aquellas con renta mensual per cápita entre 60 e 120 RS o menores a R\$ 60, considerados indigentes o extremamente pobres. De importancia en la consolidación del programa se destaca el Cadastro Único de Beneficiarios de Programas Sociales que se ha fortalecido en sus capacidades técnicas con el objetivo de ampliar el número de beneficiaros y aumentar la eficiencia al momento de la selectividad.

Durante la administración de la presidenta Rousseff fueron implementados programas adicionales. La Red Cigüeña, que buscaba ampliar la red de asistencia orientada a las mujeres embarazadas y los niños recién nacidos para reducir la mortalidad materna. Presentado como complemento del programa Bolsa Família declara como objetivo la erradicación de la pobreza extrema, para lo cual se aumenta la renta y acceso a servicios de salud y educación para 16.2 millones de personas de familias cuya renta per cápita es inferior a $\mathrm{R} \$ 70$ por mes.

La dinámica de formación de ciudadanía social comenzó con la Constitución de 1988 y las políticas de estabilidad y se consolidó por el doble movimiento de aumentos de salario mínimo y ampliación de los programas de transferencia condicionada. De hecho, el gasto en transferencia de renta para las familias pasó del $6.8 \%$ del total en 2003 a 9\% en 2012 (Singer, 2012). Así, entre 2002 y 2008 hubo una caída pronunciada del número de personas en situación de pobreza e indigencia, pasando la primera 36\% para 23\% (Singer, 2012). La combinación de aumentos de asalario mínimo y expansión de programas sociales de modo de atenuar el carácter focalizado se expresó en una reducción de la desigualdad. De acuerdo al STN, el coeficiente de Gini disminuyó del 0,5942 en 2002 al 0,5227 en 2014". (STN, 2016, p.3). ¿Significa eso que Brasil estaba en un proceso de construcción de un régimen de bienestar? Los reparos, como se mencionó para el caso argentino, se relacionan con la persistencia de núcleos duros de pobreza, alta heterogeneidad estructural, alta dispersión salarial y brechas de infraestructura y gasto social, al que se le agrega, desde el proceso de interrupción institucional, una orientación mercado céntrica de las políticas públicas.

\section{REVERSIÓN CONSERVADORA Y POLÍTICAS SOCIALES EN EL BRASIL}

Temer asumió con una agenda proclive a los sectores ortodoxo, implementando medidas liberales con el objetivo de mejor el clima de negocios, incluyendo liberalización y desregulación económica, contención del gasto público, reforma laboral e intento de reforma previsional. 
En materia de gasto público el presidente promovió una medida para limitar los gastos (e inversiones) del Estado durante un período de veinte años ${ }^{24}$, en una medida que constitucionaliza la austeridad El propósito sería reducir el gasto primario del gobierno federal del 20\% al 12\% del PIB entre 2017 e 2036, (Fagnani, 2017). La enmienda parlamentaria, que contó con respaldo de la base que participó del irregular juicio político ${ }^{25}$ y apenas con la oposición de sectores de izquierda ${ }^{26}$, promulgada el 15 de diciembre, limita el aumento que se puede hacer del gasto público en cada presupuesto a los gastos del año anterior corregidos por la inflación ${ }^{27}$, lo que redunda en un congelamiento de la inversión pública y una caída en términos per cápita. En esa línea se avanzó en un Pacto Nacional por el equilibrio de las cuentas públicas, con la presencia de los gobernadores y con el compromiso de establecer un límite de gasto para las finanzas estaduales. En primer lugar, la limitación de los gastos públicos es funcional a una estrategia de aumentar el poder del mercado en la prestación de políticas sociales. En materia de salud, existen iniciativas en marcha de manera de volver los planos de medicina privada más accesibles, lo que, en opinión de los especialistas, aumentaría la fragmentación del sistema y atentaría contra el proceso de consolidación del SUS (Vieigas et. al., 2016). En segundo lugar, es un ataque a las denominadas "vinculaciones constitucionales" para gasto en salud y educación, que han sido una construcción del sistema legal brasileño. En el caso de la educación, el artículo 2012 de la Constitución de 1988 establece la vinculación de gastos de los diferentes niveles de gobierno (Fagnani, 2017) que es de $18 \%$ de la Receita Líquida de Impostos (RLI). En cuanto a la salud, la Enmienda Constitucional 86 establecía un porcentaje de la receta corriente líquida que aumentaría de forma escalonada: 13,2\% en 2016, 13,7\% en 2017, 14,2\% en 2018, 14,7\% en 2019 e 15\% a partir de 2020.

La aprobación de la medida pone en cuestión la ampliación del gasto. Arias Vázquez (2017), utilizando datos públicos ${ }^{28}$ llevó a cabo una simulación potencial de como hubiera sido la evolución de los gastos sociales durante los años 2003-2015 en caso de haber sido regido por los parámetros de la PEC 241 recientemente aprobada, concluyendo que: i) El total de corte potencial en gasto en asistencia social sería de R\$ 437,7 mil millones, una reducción de $68,5 \%$ do gasto federal aplicado; ii) El corte en gasto en educación y cultura hubiera sido de $\mathrm{R} \$ 453,9$ mil millones, $39 \%$ a los recursos federales efectivamente invertidos; iii) El gasto en salud hubiera sido menor en $\mathrm{R} \$$ $253,4,26,5 \%$ menor; iv) el gasto en el sistema previsional hubiera sido menor en $\mathrm{R} \$ 1,7$ billones de reales, un corte de $32,6 \%$ de los recursos destinados al pago de pensiones e, v) el corte toral hubiera sido de 3.2 billones de reales en recursos asociados al gasto social, un 37\% al gasto efectivamente consolidado durante el período 20032015. Dwek e Rossi (2016) calculan fuerte reducción de la receta líquida destinada a educación (de 18,0\% a 11,3\%) y salud (de 15,0 \% para 9.3\%) hasta el año de vigencia de la Ley (2036).

En materia de regulación de las relaciones de trabajo, la coalición de gobierno impulsó una reforma laboral de tenor liberalizante ${ }^{29}$ que echa por tierra una legislación 
reguladora de las relaciones laborales. La reforma, respaldada por la comunidad empresaria ${ }^{30}$ genera cambios en el modelo político, jurídico y simbólico que reguló las relaciones entre Estado, empresarios y trabajadores durante casi 70 años. Entre los múltiples cambios que habilita el texto promulgado, se habilita la tercerización para cualquier función de la empresa (en legislación particular), aumenta la jornada de trabajo parcial de 25 a 30 horas (incluyendo la jornada intermitente que no estaba previsto en la CLT), habilita intervalos menores a una hora para almuerzo, permite la extensión de la jornada laboral diaria (supeditado a que la compensación sea durante el mismo mes y que no sea m mayor a diez horas), habilita el fraccionamiento de vacaciones en hasta tres períodos (uno de los cuales debe ser de al menos 14 días), acaba con el impuesto sindical obligatorio $y$, fundamentalmente, permite acuerdos "sobre lo legislado" en una serie de asuntos: duración de la jornada, ajuste de salarios, vacaciones, aguinaldo, banco de horas, participación en lucros y condiciones de trabajo en ambientes de baja y media insalubridad.

La reforma previsional, promovida con fuerza por la coalición de gobierno como forma de avanzar en el equilibro de las cuentas públicas una reforma de previsión social que está siendo tramitada, la PEC $28^{31}$ que elimina el derecho a una jubilación sólo por tiempo de servicio, condicionándolo a edades mínimas: 65 años para los hombres y 62 para las mujeres. Los años de contribución obligatoria aumentan de 15 a 25 años. El haber jubilatorio queda supeditado a la cantidad de años de contribución efectiva, siendo necesario 45 para tener derecho a una jubilación integral. La reforma limita el derecho de los trabajadores a acceder a una jubilación, en un contexto de alta heterogeneidad estructural. En ese sentido, Mostafa \& Theodoro (2017) afirman que la unificación de los regímenes de jubilación tenderá a reducir el tiempo medio de contribución al sistema público y estimulará la fuga de los trabajadores con mejores remuneraciones, el aumento de los años de contribución tendrá efectos sobre el grado de exclusión del beneficio previsional, teniendo en cuenta la alta informalidad, llevando a una parte de la población, estimada entre 35.5 y $40.6 \%$ a no poder jubilarse.

La propuesta del gobierno ignora que el sistema brasileño era superavitario y que en parte la falencia se debe a que el Estado puede usar hasta el $30 \%$ de los recursos recaudado por la seguridad social e inclusión por supuesto el sistema provisional para otros gastos lo que Brasil se llama como desvinculación de los recursos de la unión. En ese sentido cálculo de la ANFP declaran que los recursos retirados del sistema provisional llegaron hace 61,000 millones en 2015 y 92,000 millones en 2016 en el periodo 20082017 fueron más de 500,000 millones que fueron retirados del circuito de la Seguridad Social.

En un contexto de desmonte de las políticas heredadas del período previo (producto de la combinación de suspensión de beneficios, aumentos por debajo de la inflación que lleva a pérdida del -escaso- poder adquisitivo de las transferencias o inclusión de mayores requisitos para ser protegido) el gobierno implementó un programa denominado Progresar (Progredir) que tenía como objetivo declarado 
generar empleo y renta, específicamente, para las familias registradas en el Cadastro Único de Programas Sociales y en el Programa Bolsa Família. Presentado de manera ambiciosa como un programa de inclusión productiva que ofrecería un millón de puestos en cursos de formación profesional, el programa cumplió las metas (capacitó, según el Ministerio de Desarrollo Social, 1.047.359 vagas, pero lo hizo con cursos a distancia, sin mayor impacto en las tasas de empleo del sector que se buscaba beneficiar.

El gobierno Temer creó el programa Niño Feliz ( Criança Feliz) con el objetivo declarado de proteger a la primera infancia, desde el nacimiento hasta los 6 años de vida. A pesar de que, al momento de lanzarlo, el gobierno declaraba buscar proteger 4 millones de niños de hasta tres años, el programa estuvo lejos de cumplir sus objetivos, protegiendo 166.378 niños en 2017 y 190.330 en 2018, totalizando 356.708 niños en 2018. El programa recibiría 1000 millones de reales. Datos del gobierno informan que los recursos efectivamente implementados fueron sustancialmente inferiores: 435 millones de reales.

La situación social, producto de la profunda caída de la actividad, muestra un serio declive de los indicadores. Los niveles de empleo empeoraron. Los últimos datos de la Pesquisa Nacional de Amostra Domiciliar elaborada por el IBGE (abril de 2019) indica que el desempleo es de $12.7 \%$ y la subutilización (desocupados, subempleados o que están desalentados a buscar empleo), 25\%. Así, hay 13.4 millones de desempleados en el país y 28.3 millones "subutilizados", de los cuales 4.8 millones son desalentados. Entre 2016 y 2017 aumentó el número de personas viviendo bajo la línea de pobreza en el país, que pasaron de $25.7 \%$ a $26.5 \%$. La extrema pobreza pasó de $6.6 \%$ a $7.4 \%$, de 13.5 millones a 15.2 millones de personas.

La gestión Bolsonaro consolidó el carácter residual de las políticas del sistema de protección social. Eliminó el Ministerio de Desarrollo Social e instituyó un Ministerio de Ciudadanía; avanzó en la extinción de órganos colegiados por medio del Decreto 9759/19, que pone en cuestión más de 700 decretos $^{32}$ con el intento de desburocratizar el Estado, atacando la política Nacional de Participación social y el Sistema Nacional de Participación Social.

\section{A MODO DE CONCLUSIÓN}

En este breve ensayo hemos presentado las principales medidas de protección social que se implementaron en el periodo post-neoliberal en Argentina y Brasil y recuperado algunos elementos de ruptura a partir de la transición política que se produjo en ambos países (por elecciones en Argentina, por un proceso de juicio político de dudosa legitimidad en el Brasil). Ambos países han atravesado por un proceso de ampliación del gasto en protección social, a pesar de las diferencias en la selectividad, más centrado en personas en situación de pobreza en el Brasil, más centrado en la figura de trabajadores desocupados en el caso argentino. 
Durante el período de debilitamiento del neoliberalismo se observa una recuperación del papel del Estado en la protección social, quebrando con la lógica residual y minimalista que imperó en durante el período neoliberal. Hubo una mejora de la situación de la pobreza y la indigencia, producto de la combinación virtuosa entre recuperación de la actividad económica y adopción de mecanismos tendientes a mantener elevada la demanda agregada y la protección social. Las limitaciones de los esquemas de protección desplegados durante el período post-neoliberal se potenciaron a partir de la transición gubernamental que implicó, de diferentes maneras, la llegada al poder de coaliciones identificadas con la primacía del mercado como asignador de recursos y orientador de políticas. Pese a las diferencias, en ambos países se observa una primacía del mercado, una gramática que articula protección a empleo, lo que genera que la protección a la pobreza sea vista como transitoria, hasta que haya una inserción de la persona en el mercado de trabajo o su conversión en emprendedor, en consonancia con teorías de capital humano.

\footnotetext{
Notas

${ }^{1}$ Aun cuando es discutible que el país haya tenido un Estado de Bienestar en sentido estricto, existe amplio consenso en afirmar que la combinación de pleno empleo y políticas sociales cuasi-universales garantizaban movilidad social ascendente y niveles de igualdad social altos comparados con los parámetros de la región latinoamericana, por el carácter pionero da la gestación de su sistema de pensiones y beneficios sociales y una alta cobertura del sistema de protección (Golbert, 2010; Mesa Lago, 2004).

2 Las Oficinas de Empleo ofrecen atención personalizada, acompañamiento permanente, orientación laboral, información actualizada sobre el mercado laboral local a través de servicios específicos que facilitan el acceso a cursos de orientación y formación profesional gratuitos, programas de empleo, asistencia a microemprendedores, talleres para la búsqueda de empleo y vinculación con puestos de trabajo. Ver: http://www.trabajo.gov.ar/empleo/mapa_oe.asp. Acesso em: 30 oct. 2019.

${ }^{3}$ Para permanecer en el Programa Familias, las condicionalidades se verificaban en forma cuatrimestral e incluían, en el área de salud, el cumplimiento de Plan Nacional de Vacunación para los hijos menores a cargo, conforme las indicaciones del Ministerio de Salud de la Nación y controles bimestrales de para las mujeres embarazadas; en materia de educación, la matriculación inicial y continuidad de condiciones de alumnos/as regulares, para cada niño/a a cargo del titular, desde los 5 años de edad hasta los 19 años, o la culminación del nivel Secundario o Polimodal.

${ }^{4}$ Chequeado.com, Qué es la Plata de los Jubilados. http://chequeado.com/el-explicador/ique-es-la-platade-los-jubilados. Acceso en: 10 Jul. 2019.

${ }^{5}$ Entrevista a Diego Bossio, Miradas al Sur, 13/4/2018, Cada peso que ingresa al ANSES se distribuye entre los más necesitados. Consultar: http://www.miradasalsur.com.ar/2015/04/13/revista/cada-peso-queingresa-al-anses-se-distribuye-entre-los-mas-postergados. También: www.fgs.anses.gob.ar. Acceso em: 15 jun. 2017.

619 Rodríguez Use, Jerónimo. Discusión Obsoleta. IN: Página 12. El Entramado del Mercado Laboral, 20/4/2015. http://www.pagina12.com.ar/diario/economia/2-270924-2015-04-20.html. Acceso em: 15 Jun. 2019.

${ }^{7}$ Evolución del salario mínimo en Argentina durante el Kirchnerismo. http://www.latdf.com.ar/2014/09/ evolucion-del-salario-minimo-de.html. Acesso em: 15 Jun. 2019.

${ }^{8}$ Consultar: http://www.pagina12.com.ar/diario/elpais/1-139400-2010-02-01.html. Acesso em: 15 Jun. 2019

9 La implementación de la AUH, contrariando la postura que había sostenido la coalición de gobierno durante los años previos, formó parte de una radicalización más amplia del activismo estatal para sostener
} 
los niveles de demanda agregada frente a la complejidad del contexto externo. La crisis internacional, combinada con un conflicto interno desatado entre el gobierno y la burguesía industrial, representó un punto de inflexión que abrió ventanas de oportunidad para aumentar el activismo estatal en materia de políticas sociales y productivas. En ese sentido, reforzando las políticas contracíclicas el PEN lanzó diferentes programas. En julio de 2009 se pone en marcha el Programa Jóvenes por Más y Mejor Trabajo orientado a jóvenes de entre 18 y 24 años con el objetivo "generar oportunidades de inclusión social y laboral de los jóvenes, a través de acciones integradas, que les permitan construir el perfil profesional en el cual deseen desempeñarse, finalizar su escolaridad obligatoria, realizar experiencias de formación y prácticas calificantes en ambientes de trabajo, iniciar una actividad productiva de manera independiente o insertarse en un empleo". También en 2009 fue relanzado el REPRO, el Programa de Recuperación Productiva, creado por la Resolución del Ministerio de Trabajo, Empleo y Seguridad Social № 481/02 en el marco de la Emergencia Ocupacional Nacional y que volvió a tomar fuerza en 2009, con el telón de fondo de la crisis capitalista del centro económico, con sucesivas prórrogas de vigencia. El programa brinda a los trabajadores de las empresas adheridas una suma fija mensual no remunerativa por un plazo de hasta 12 meses, destinada a completar el sueldo de su categoría laboral, mediante el pago directo por ANSES.

${ }^{10}$ El Régimen Simplificado para Pequeños Contribuyentes "Monotributo Social", se regula a través del Decreto No 189/2004, Ley № 25.865 modificada mediante Decreto № 82/2004, y Ley № 26.223, Ley (2009) y Decreto No 204/2004 (MDS, 2010).

${ }^{11}$ Ver: https://www.anses.gob.ar/informacion/movilidad-de-jubilaciones-y-pensiones. Acceso en: 15 jun. 2019.

${ }^{12}$ A partir de la aplicación de la ley de movilidad las jubilaciones y pensiones aumentaron 5,71\% en marzo de 2018, 5,70\% en junio, 6,67\% en septiembre y 7,75\% en diciembre. En 2019 aumentaron 11,85\% en marzo y $10,74 \%$ en junio (CEPA, 2019).

${ }^{13}$ La inflación acumulada en los tres años de gobierno de Cambiemos (excluyendo 2019) es superior al 100\%: 33,7\% en 2016, 24,8\% en 2017 y 47,6\% en 2018.

${ }^{14}$ A comienzos de 2018 el presidente Macri eliminó el rango ministerial del área de trabajo, que pasó a ser uma Secretaría dependiente del Ministerio de Producción.

${ }^{15}$ Consultar: https://bit.ly/2pqvmFp. Acceso en 17 Jun. 2019.

${ }^{16}$ Consultar: https://bit.ly/2Ir1Peh. Acceso en 23 Jun. 2019.

17 Consultar: https://bit.ly/2mCfcbL. Também: http://www.infobae.com/politica/2017/06/15/la-nuevacupula-de-la-uia-se-reunio-con-mauricio-macri-y-coincidio-en-enfrentar-la-mafia-de-los-juicioslaborales/. Y también: http://www.infocampo.com.ar/la-sra-y-fadeeac-salieron-a-apoyar-a-macri-sobrela-mafia-de-los-juicios-laborales/. Acceso en 16 Jun. 2019.

18 Ver: https://internacional.elpais.com/internacional/2017/04/12/argentina/1492003488_924163.html. Acceso en: 15 Jun. 2019.

${ }^{19}$ Un error en el cálculo de los haberes previsionales que redujo los valores, una suspensión en el pago de las becas para estudiantes de enseñanza media, una suspensión de miles de pensiones asistenciales a personas con discapacidad figura en el listado de "errores" subsanados, parcialmente, luego de ser denunciados.

20 "Tenemos una situación de emergencia que atender, la primera manera de hacerlo es que aquellas personas que no tienen un trabajo digno reciban recursos del Estado", sostuvo. Stanley dijo: "Todos queremos que los argentinos tengan un empleo de calidad, pero mientras tanto necesitamos garantizarles un ingreso, como con la asignación por hijo o la pensión por vejez". Ver: http://www. diariobae.com/article/details/107214/la-pobreza-el-asistencialismo-estatal-y-el-compromiso-social-enel-centro-del-de. Acceso en: 16 Jun. 2019.

${ }^{21}$ Em septiembre de 2003 el programa Bolsa-Escola alcanzaba a cerca de cinco millones de famílias, el Bolsa-Alimentação aproximadamente 1,6 millón de famílias, el Auxílio-Gás, 9,7 millones de famílias y el Cartão-Alimentaçãoa algo menos de 775 mil.

${ }^{22}$ La implementación del PBF estuvo a cargo de la Secretaria Nacional de Renda e Cidadania (Senarc), del Ministério do Desenvolvimento Social e Combate à Fome (MDS) (Bichir, 2016).

${ }^{23}$ Brasil cuenta desde 2005 con una ley de renta básica de ciudadanía que, a pesar de no estar implementada, puede ser considerada un faro para las políticas que fueron implementadas.

${ }^{24}$ Ver: https://www.cartacapital.com.br/revista/920/temer-a-pec-241-e-a-entrega-irrestrita-ao-neoliberalismo. Acceso en: 15 jul. 2019.

${ }^{25}$ http://www.resumenlatinoamericano.org/2016/10/11/brasil-tres-duras-medidas-de-temer-muestranla-verdadera-cara-del-golpe-neoliberal-congelan-el-gasto-publico-por-20-anos. Acceso en: 15 jul. 2019. 


\begin{abstract}
${ }^{26}$ PT, PDT, Rede, PSOL, PCdoB, PMB.
${ }^{27}$ Quedaron fuera de las nuevas reglas, entre otras, las trasnferencias constitucionales a las unidades fedreativas (los Estados), los créditos extraordinários, los gastos de la justicia electoral com elecciones, los gastos com capitalizaciones de empresas estatales.

${ }^{28}$ Ministério da Fazenda sobre o "Gasto Social do Governo Central - 2002 a 2015" (STN, 2016).

${ }^{29}$ Consultar: http://www.bbc.com/portuguese/brasil-40577806; https://bit.ly/30FvD5X. Também: https://bit.ly/2ZjRIQx. Acceso en: 15 jul. 2019.

${ }^{30}$ Consultar: https://theintercept.com/2017/04/26/lobistas-de-bancos-industrias-e-transportes-quemesta-por-tras-das-emendas-da-reforma-trabalhista. Acceso en: 15 jul. 2019.

31 Consultar: http://www1.folha.uol.com.br/mercado/2017/05/1881011-veja-as-principais-mudancas-naproposta-de-reforma-da-previdencia.shtml. Acceso en: 15 jul. 2019.

32 Em palabras del Jefe de la Casa Civil Onyx Lorenzoni, la ntención es reducir el número de órganos colegiados de 700 a 50.
\end{abstract}

\title{
Bibliografía
}

ABERS, R.; SERAFIM, L. \& TATAGIBA, L. (2014). Repertórios de interação estado-sociedade em um estado heterogêneo: a experiência na Era Lula. Dados, v.57, n.2, pp.325-357.

ALVES GUTIERRES, K. (2015). Projetos políticos, trajetórias e estratégias. A política de assistência social entre o partido e o Estado. PhD dissertation, State University of Campinas, 2015.

ANTÍA, F. (2018). Regímenes de política social en América Latina: una revisión crítica de la literatura. Desafíos, v. 30, n. 2, Julio-Diciembre, pp. 193-235 Universidad del Rosario Bogotá, Colombia

ANSES. (2011). Inclusión y previsión social en una Argentina responsable. Informes de prensa.

BORGES SUGIYAMA, N. (2011). The diffusion of conditional cash transfer programs in the Americas. Global Social Policy, n. 11(2-3), 250-278.

BORGES SUGIYAMA, N. (2012). The Diffusion of Good Government: Social Sector Reforms in Brazil. Notre Dame, IN: University of Notre Dame Press.

BOSCHI, R. \& GAITAN, F. (2016). Elites, coalizões e Desenvolvimento. Análise sobre a trajetória recente do Brasil. Desenvolvimento em Debate. v.4, n.2, p.29-61

DANANI, C.; BECCARIA, A. (2011). La (contra)reforma previsional argentina 2004-2008: aspectos institucionales y político-culturales del proceso de transformación de la protección. In: Danani, C.; Hintze, S. (Org). Protecciones y desprotecciones: la seguridad social en la Argentina, 1990-2010. Buenos Aires: UNGS. p. 103-151.

DE LA O, A. (2013). Do conditional cash transfers affect electoral behaviour? Evidence from a randomized experiment in Mexico. American Journal of Political Science, v57, n1, p. 1-14.

DESAI, Raj. The political economy of poverty reduction. Scaling up antipoverty programs in the Developing world. Wolfhenson Centre for Development. Brooking Institution, 2007.

DION, M. L. (2009). Workers and welfare: Comparative institutional change in twentieth-century Mexico. Pittsburgh: University of Pittsburgh Press.

ECLAC. (2017). Social Panorama of Latin America. Santiago: ECLAC.

ETCHEMENDY, S. (2017). The Politics of Popular Coalitions in Post-Neoliberal Latin America (2000-2015). Paper submitted to the 9th Congress of the Latin American Political Science Association (ALACIP), Montevideo July 26-28. 
FILGUEIRA, F. (2005). Welfa re and democracy in Latin America: The development, crises and aftermath of universal, dual and exclusionary social States. Preparado para el proyecto en política social y democratización, UNIRSD.

FRANCO, R. (1996). Los paradigmas de la política social en América Latina. Revista de la CEPAL, $\mathrm{n}$. 58, p. 9-22.

GARAY, C. (2016). Social policy expansión in Latin America. New York: Cambridge University Press. GOLBERT, L. (2004). Derecho a la inclusión o paz social? El Plan Jefas y Jefes de Hogar Desocupados, Santiago: CEPAL, 2004.

HAAS, P. (1992). Introduction to epistemic communities and International Policy Coordination. International Organization. Vol. 46 n¹, Knowledge, Power, and International Policy Coordination, 1-35.

HAGGARD, S. \& KAUFMAN, R. (2008). Development, democracy, and welfare states: Latin America, East Asia, and Eastern Europe, Princeton: Princeton University Press.

HALL P. (1992). The movement from Keynesianism to monetarism: institutional analysis and British economic policy in the 1970s. New York: Cambridge Univ. Press.

HANLON, J.; BARRIENTOS, A. \& HULME, D. (2010). Just Give Money to the Poor: The Development Revolution from the Global South. Sterling, VA, USA: Kumarian.

HUBER, E. \& STEPHENS, J.D. (2012). Democracy and the left: Social policy and inequality in Latin America. Chicago: The University of Chicago Press.

IPEA (2012). Políticas sociais: acompanhamento e análise. Brasília: IPEA.

KING, G.; KEOHANE, R.; Verba, S. (1994). Designing social inquiry: scientific inference in qualitative research. Princeton: Princeton University Press.

MAHONEY, J. (2010). After KKV: the new methodology of qualitative research. World Politics, v. 62 , n. 1, p. 120-147.

MARES, I. \& CARNES, M. (2009). Social Policy in Developing Countries. Annual Review of Political Science, n. 12, p. 93-113.

Ministerio de Desarrollo Social (2010). Rendimos Cuentas, Buenos Aires: MDS.

MIRÁNDOLA BICHIR, R. (2016). A emergência e a consolidação de programas de transferência de renda no Brasil, na Argentina e na África do Sul. In: Gomide, Alexandre \& Boschi, Renato. Capacidades Estatais em Países Emergentes: o Brasil em perspectiva comparada. Brasília: IPEA.

NIEDZWIECKI, S. (2016). Social policies, attribution of responsibility and political alignments. A subnational analysis of Argentina and Brasil. Comparative Political Studies, v. 49, issue 4.

PALOMINO, H.\& TRAJTEMBERG, D. (2006). Una nueva dinámica de las relaciones laborales y la negociación colectiva en la argentina. Revista de Trabajo, 2(3): 47-68.

PRIBBLE, J. (2013). Welfare and Party Politics in Latin America. Cambridge, Cambridge University Press.

PRIBLE, J.; HUBER, E. \& STEPHENS, J. (2009). The politics of effective and sustainable redistribution. Washington DC: Brooking Institution.

PRZEWORSKI, A.; TEUNE, H. The Logic of Comparative Social Inquiry. New York: John Wiley \& Sons, 1970.

ROSSI, F. M. The second wave of incorporation in Latin America: A conceptualization of the quest for inclusion applied to Argentina. Latin American Politics and Society, 57(1), 1-28, 2015.

SEGURA-UBIERGO, A. The political economy of the welfare state in Latin America: Globalization, democracy, and development. Cambridge: Cambridge University Press, 2007. 
SILVA, S. (2018). Democracia, políticas publicas e instituições de deliberação participativa: visões sobre a experiência brasileira. Texto de Discussão IPEA 2358.

SOARES, S. (2012). Bolsa Familia, its design, its impacts and possibilities for the future. Working Paper N. 89, Institute Policy Center for the Future, Feb.

SOUZA ALVARENGA, M. \& TEIXEIRA GARCIA, M. L. (2015). O processo de elaboração da política nacional de assistência social de 2004. Textos e Contextos, v. 14, n. 1, p. 202-214.

ARCIDIÁCONO, Piular, GAMALLO, Gustavo \& STRACHNOV, Mora. Programas sociales y protección social no contrituvia em la post-convertibilidad argentina, Revista de Instituciones, IDeas y Mercados, N 60, mayo 2014, pp. 57-86.

DEL VALLE, Alejandro \& BODA DANTE, Jeremías. Protección social y protecciones de las políticas sociales en Argentina. Textos \& Contextos (Porto Alegre), v. 14, n. 2, pp. 338-358,agosto dezembro 2015.

TONELLI Vaz, Flávio. Reforma da previdência de Bolsonaro: excluindo os pobres do orçamento, Asesoria Técnica, Liderança do PCDB.

MARQUES, Rosa Maria. Permanência e ruptura na proteção social brasileira no período recente. Argumentum, Vitória, v. 11, N. 1, PP. 130-145, janeiro-abril 2019. 